\title{
A NONLINEAR COMPLEMENTARITY PROBLEM IN MATHEMATICAL PROGRAMMING IN BANACH SPACE
}

\author{
M. S. BAZARAA, J. J. GOODE AND M. Z. NASHED
}

\begin{abstract}
An existence and uniqueness theorem for the nonlinear complementarity problem over closed convex cones in a reflexive real Banach space is established, using perturbations of solutions of variational inequalities and monotonicity methods.
\end{abstract}

1. Introduction and statement of the main result. Let $B$ be a reflexive real Banach space and let $B^{*}$ be its topological dual. Let the value of $u \in B^{*}$ at $x \in B$ be denoted by $\langle x, u\rangle$. Let $C$ be a closed convex cone in $B$ with vertex at 0 and polar cone $C^{*}$, i.e., $C^{*}=\left\{u \in B^{*}:\langle x, u\rangle \leqq 0\right.$ for each $x \in C\}$. A mapping $T: C \rightarrow B^{*}$ is said to be monotone if $\langle x-y, T x-T y\rangle \geqq 0$ for all $x, y \in C$, and strictly monotone if the strict inequality holds whenever $x \neq y$. We shall also say that $T$ is $\alpha$-monotone if there is a strictly increasing function $\alpha:[0, \infty) \rightarrow[0, \infty)$ with $\alpha(0)=0$ and $\alpha(r) \rightarrow \infty$ as $r \rightarrow \infty$ such that $\langle x-y, T x-T y\rangle \geqq\|x-y\| \alpha(\|x-y\|)$ for each $x, y \in C$. In particular, $T$ is strongly monotone if $\alpha(r)=k r$ for some $k>0$. $T$ is said to be hemicontinuous on $C$ if for any $u, v \in C$, the map $t \rightarrow T(t v+(1-t) u)$ of $[0,1]$ to $B^{*}$ is continuous when $B^{*}$ is endowed with the weak * topology. $T$ is said to be demicontinuous on $C$ if $u_{n} \rightarrow u$ in the strong topology implies that $T u_{n}$ converges to $T u$ in the weak* topology of $B^{*}$. See Kato [2] and Minty [3] for properties of monotonicity, demicontinuity, and hemicontinuity.

The purpose of this note is to prove the following existence and uniqueness theorem for the nonlinear complementarity problem which encompasses a variety of problems arising in mathematical programming and optimization theory.

Received by the editors March 19, 1971.

AMS 1970 subject classifications. Primary 46N05, 47H15, 90C99: Secondary 47H05, $90 \mathrm{C} 30$.

Key words and phrases. Nonlinear complementarity problem, operator equations with constraints, perturbations of variational inequalities, convex cones, nonlinear programming, monotone operators.

1 Research of this author was sponsored by the Mathematic Research Center, under contract number DA-31-124-AR()-I)-462 with the United States Army. 
THEOREM. Let $T: C \rightarrow B^{*}$ be a bounded hemicontinuous, $\alpha$-monotone operator. Then there exists a unique $x_{0} \in C$ such that $-T x_{0} \in C^{*}$ and $\left\langle x_{0}, T x_{0}\right\rangle=0$.

For a thorough treatment of the nonlinear complementarity problem in finite dimensional spaces with a particular choice of the cone $C$, we refer to Karamardian [1], where the problem is also related to nonlinear programming, saddle point theory, and the equilibrium point problem of an $n$-person game.

We approach the nonlinear complementarity problem in Banach space from the viewpoint of continuous dependence of the solutions of the variational inequality

$$
x \in K:\langle x, T x\rangle \leqq\langle z, T x\rangle \text { for all } z \in K,
$$

where $T$ is a fixed strictly monotone operator on the perturbations of the set $K$. This turns out to be an efficient approach to the nonlinear complementarity problem which seems to have been overlooked even for the finite dimensional version of the problem (see also Remark 2 in $\S 3)$. This framework allows us also to admit perturbations in the operator $T$ and to replace $T$ by a sequence of maps $T_{n}$ (possibly with a finite dimensional range) which approximate $T$. Thus, this approach lends itself more readily to computational aspects of the nonlinear complementarity problem, although we shall not be concerned with this here.

2. Proof of the Theorem. For each $p>0$, let $S_{p}=\{x \in B:\|x\| \leqq p\}$ and $K_{p}=C \cap S_{p}$. We shall need a notion describing the convergence of $K_{p}$ to $K_{p_{0}}$ as $p \rightarrow p_{0}$ for $p, p_{0} \in(0, \infty)$. To this end, we use the following notation in which strong lim denotes convergence in the strong topology of $B$, while weak lim denotes convergence in the weak topology of $B$.

$$
\begin{aligned}
& \mathrm{s}-\liminf _{p \rightarrow p_{0}} K_{p}=\left\{v \in B: v=\text { strong limit of } v_{p} \text { as } p \rightarrow p_{0},\right. \\
& \left.\qquad v_{p} \in K_{p} \text { for each } p>p_{0}\right\},
\end{aligned}
$$

$\mathrm{w}-\lim \sup K_{p}=\left\{v \in B: v=\right.$ weak limit of $v_{j}$ as $j \rightarrow \infty, v_{j} \in K_{p_{j}}$ for

$$
\text { every integer } \left.j, p_{j} \rightarrow p_{0} \text { as } j \rightarrow \infty\right\} \text {. }
$$

Following Mosco [4] we say that $\left\{K_{p}\right\}$ converges in $B$ if the two sets above coincide, and we write $K_{p_{0}}=\lim _{p \rightarrow p_{0}} K_{p}$ in $B$ if $K_{p_{0}}$ is a subset of $B$ such that

$$
\mathrm{s}-\lim _{p \rightarrow p_{0}} \inf K_{p}=\underset{p \rightarrow p_{0}}{\mathrm{w}-\lim \sup _{p}} K_{p}=K_{p_{0}} .
$$

Noting that $K_{p} \subset K_{q}$ if $p<q$ and that $K_{p}$ is a nonempty closed convex set for each $p \in(0, \infty)$ it is easy to show that if $p \rightarrow p_{0} \in(0, \infty)$, then $K_{p} \rightarrow K_{p_{0}}$ 
in the sense of convergence just introduced. (This alsu follows from the more general setting of Lemmas 1.2 and 1.3 in Mosco [4].) We shall need the following special case of Theorem A of Mosco [4].

Lemma 1. Let $T: C \rightarrow B^{*}$ be a bounded hemiconıinuous and strictly monotone operator and let $\left\{K_{p}\right\}$ be a family of nonempty closed convex sets in $C$. Then each of the variational problems

$$
x_{p} \in K_{p}:\left\langle x_{p}, T x_{p}\right\rangle \leqq\left\langle z, T x_{p}\right\rangle \text { for all } z \in K_{p}
$$

has a unique solution. If in addition $K_{p} \rightarrow K_{p_{0}}$ and $T$ is $\alpha$-monotone, then the mapping $p \rightarrow x_{p}$ is continuous at $p_{0}$ with the strong topology on $C$.

Let the function $\gamma:[0, \infty) \rightarrow(-\infty, \infty)$ be defined by $\gamma(p)=\left\langle x_{p}, T x_{p}\right\rangle$. We note that if $T$ is demicontinuous and $\alpha$-monotone, then the function $\gamma$ is continuous. If in addition $-T(0) \notin C^{*}$, then there is $p_{0}>0$ such that $\gamma\left(p_{0}\right)<0$.

Lemma 2. If $T: C \rightarrow B^{*}$ is a hemicontinuous and strictly monotone operator, and $x_{p}$ is the unique solution of (1) in $K_{p}=C \cap S_{p}$, then either $\left\langle x_{p}, T x_{p}\right\rangle=0$ or $\left\|x_{p}\right\|=p$.

Proof. If $\gamma(p) \neq 0$, then $x_{p} \neq 0$, and $\left(p /\left\|x_{p}\right\|\right) x_{p} \in K_{p}$. Hence

$$
\gamma(p)=\left\langle x_{p}, T x_{p}\right\rangle \leqq\left\langle\left(p /\left\|x_{p}\right\|\right) x_{p}, T x_{p}\right\rangle=\left(p i\left\|x_{p}\right\|\right) \gamma(p) .
$$

Since $0 \in K_{p}$, it is clear that $\gamma(p) \leqq 0$. Thus $\gamma(p) \neq 0$ implies that $\left\|x_{p}\right\| \geqq p$, and hence $\left\|x_{p}\right\|=p$ since $x_{p} \in K_{p}$.

Proof of the Theorem. First note that the system

$$
x \in C, \quad-T x \in C^{*}, \quad\left\langle x, T x_{i}=0\right.
$$

has at most one solution if $T$ is strictly monotone. For if $x_{1}$ and $x_{2}$ are two solutions, then $x_{1}, x_{2}$ are both in $C,-T x_{1}$ and $-T x_{2}$ are both in $C^{*}$ and thus $\left\langle x_{1}, T x_{2}\right\rangle \geqq 0$ and $\left\langle x_{2}, T x_{1}\right\rangle \geqq 0$. But we also have $\left\langle x_{1}, T x_{1}\right\rangle=$ $\left\langle x_{2}, T x_{2}\right\rangle=0$, and therefore $\left.\left\langle x_{1}-x_{2}, T x_{1}-T x_{2}\right\rangle=x_{1}, T x_{1}\right\rangle+\left\langle x_{2}, T x_{2}\right\rangle-$ $\left.\left\langle x_{1}, T x_{2}\right\rangle-x_{2}, T x_{1}\right\rangle \leqq 0$. If $T$ is strictly monotone we conclude that $x_{1}=x_{2}$. Clearly if $T$ is $\alpha$-monotone, then $T$ is strictly monotone.

Now note that if $\gamma(p)=0$ for some $p$, then $0=\left\langle x_{p}, T x_{p} \leqq\left\langle z, T x_{p}\right\rangle\right.$ for each $z \in K_{p}$. Hence also $\left\langle z, T x_{p}\right\rangle \geqq 0$ for each $z \in C$ and thus $x_{0}=x_{p}$ is the unique solution to the system (2). We now show that when $T$ is $\alpha$-monotone, it is impossible to have $\gamma(p) \neq 0$ for all $p$. Since $0 \in K_{p}$ it is clear that $\gamma(p) \leqq 0$. Thus $\gamma(p) \neq 0$ implies that $\gamma(p)<0$ for all $p$. Take $p>1$. Then $\gamma(1)=\left\langle x_{1}, T x_{1}\right\rangle \leqq\left\langle z, T x_{1}\right\rangle$ for all $z \in K_{1}$ and $\left\langle x_{p}, T x_{p} \leqq z, T x_{p}\right\rangle$ for all 
$z \in K_{p}$. Noting that $p x_{1} \in K_{p}$ and $p^{-1} x_{p} \in K_{1}$, we have $p \gamma(1) \leqq\left\langle x_{p}, T x_{1}\right\rangle$ and $p^{-1} \gamma(p) \leqq\left(x_{1}, T x_{p}\right)$. Thus

$$
\begin{aligned}
{\left[p^{-1} \gamma(p)-\right.} & \gamma(1)](p-1) \\
& \left.\left.=x_{n} . T x_{p}-p^{-1}\left\langle x_{p}, T x_{p}\right\rangle-p x_{1}, T x_{1}\right\rangle+x_{1}, T x_{1}\right\rangle \\
& \left.\geqq x_{p}, T x_{p}\right\rangle\left\langle x_{1}, T x_{n}\right\rangle-\left\langle x_{p}, T x_{1}\right\rangle+x_{1}, T x_{1} \\
& \left.=x_{p}-x_{1}, T x_{p}-T x_{1}\right\rangle .
\end{aligned}
$$

Then since $T$ is $\alpha$-monotone and $p>1$, we have

$$
p^{-1} \gamma^{\prime}(p) \geqq i^{\prime}(1)+(p-1)^{-1}\left\{\left\|x_{p}-x_{1}\right\| \alpha\left(\| x_{p}-x_{1} i i\right)\right\} .
$$

Now since $\gamma(p) \neq 0$ and $\gamma(1) \neq 0$, we have using Lemma $2,\left\|x_{p}--x_{1}\right\| \geqq$ $\left\|x_{p}\right\|-\left\|x_{1}\right\|=p-1$. Thus $p^{-1} \gamma(p) \geqq \gamma(1)+\alpha(p-1)$. Since $\alpha(r) \rightarrow \infty$ as $r \rightarrow \infty$, the last inequality implies that there exists a $p$ such that $\gamma(p)>0$. This contradiction implies that the assumption $\gamma(p) \neq 0$ for all $p$ is impossible. We have in fact shown that $p^{-1} \gamma(p)$ is a strictly increasing function of $p$ until $\gamma(p)=0$; for larger values of $p, x_{p}$ is a constant vector and $\gamma(p)=0$.

\section{Remarks.}

REMARK 1. The origin and the name of the complementarity problem go back to the following programming problem: Given a vector $b$ in $R^{n}$ and a real $n \times n$ matrix $M$, find a vector $x=\left(x_{1}, \cdots, x_{n}\right)$ such that $x_{i} \geqq 0, y=M x+h, y_{i} \geqq 0$, and $x_{i} y_{i}=0$ for $i=1, \cdots, n$. Each pair $\left(x_{i}, y_{i}\right)$ is called a complementary pair. This problem is quite general and includes, by appropriate choice of the vector $b$ and the matrix $M$, a variety of problems in linear and quadratic programming, game theory, etc. (see [1] for a detailed discussion and references to earlier contributions to the linear complementarity problem.). Karamardian [1] considered the following problem: Given a function $f: R^{n} \cdots R^{n}$, find a vector $x \in R^{n}$ which satisfies the system $x \geqq 0, f(x) \geqq 0, x \cdot f(x)=0$, where the ordering is componentwise. The main result in [1] is that this problem has a unique solution if $f$ is continuous and strongly monotone on the nonnegative orthant $R_{+.}^{n}$ of $R^{n}$. Our theorem gives a more general result when specialized to finite dimensional spaces since we replace the nonnegative orthant by an arbitrary closed convex cone with vertex at the origin and slightly weaken the monotonicity hypothesis on $f$.

REMARK 2. Our first attempt to prove an existence and uniqueness theorem for the nonlinear complementarity problem in Banach space was essentially to generalize the arguments in [1] via an infinite dimensional analogue of the Kakutani fixed point theorem for multivalued mappings, such as the Bohnenblust-Karlin theorem or recent fixed point theorems of F. F. Browder and Ky Fan. This has failed since in generalizing Lemmas 2.1 2.2 and the proof of Theorem 2.1 in [1] we had to 
impose that the mapping is completely continuous and strongly monotone; this implies that the inverse of the mapping is bounded which, together with complete continuity, puts us back in the finite dimensional space. The technique of using perturbations of solutions of variational inequalities, which does not seem to have been explored in the context of mathematical programming, merits further consideration.

REMARK 3. In many problems where the nonlinear complementarity problem arises, the operator $T$ is a gradient mapping, i.e., there exists a Gâteaux differentiable functional $g: C \rightarrow R$ such that

$$
\left.(d / d t) g(u+t h)\right|_{t=0}=\langle h, T u\rangle \text { for } h \in B, u \in C .
$$

In this case, we may use the characterizations given in the following proposition of the properties of monotonicity and $\alpha$-monotonicity of $T$ in terms of appropriate convexity of $g$. We call a function $g: C \rightarrow R \alpha$ convex if and only if there exists a function $\alpha:[0, \infty) \rightarrow[0, \infty)$ with the properties stated in $\S 1$ such that for all $t \in[0,1]$,

$$
\begin{aligned}
& g(t u+(1-t) v) \leqq t g(u)+(1-t) g(v) \\
&-\|u-v\| \max \{t \alpha[(1-t)\|u-v\|],(1-t) \alpha[t\|u-v\|]\} .
\end{aligned}
$$

Thus the usual notion of a convex functional corresponds to taking $\alpha$ to be the zero function.

Proposition. Let $g$ be a real-valued functional on an open convex subset $\Omega$ of a normed real space $E$. Let $g$ have a Gâteaux derivative which is continuous on line segments in $\Omega$. Then:

(a) $g$ is convex on $\Omega$ if and only if grad $g$ is a monotone operator;

(b) $g$ is strictly convex if and only if grad $g$ is strictly monotone;

(c) $g$ is $\alpha$-convex if and only if grad $g$ is $x$-monotone: and

(d) $\operatorname{grad} g$ is strongly monotone if and only if $g$ is $\alpha$-con's $x$ with $\alpha(t)=m t$ and $m>0$.

This proposition follows easily using simple manipulations based on the relation

$$
g(u)-g(v)=\int_{0}^{1} u-v, \operatorname{grad} g(t t+(1-t) u) d t .
$$

Added IN PROOF (MAY 23, 1972). Since the submission of this paper several papers have been written about the nonlinear complementarity problem. Among those which are related to this paper we mention the following: G. J. Habetler and A. L. Price, Existence theory for generalized nonlinear complementarity problem. J. Gptimization Theory Appl. 7 (1971), 223-239; B. C. Eaves, On the hasic theorem of complementarity, 
Math. Programming 1 (1971), 68-75; S. Karamardian, The complementarity problem, Math. Programming 2 (1972), 107-129; and S. Karamardian, The generalized complementarity problem, J. Optimization Theory Appl. (to appear). In some of these papers the complementarity problem is also studied with the usual nonnegative partial ordering of $R^{n}$ replaced by partial ordering induced by a given cone and its polar; the equivalence of the complementarity problem to a variational inequality is also observed. While the existence and uniqueness of the solution of the complementarity problem can be obtained without recourse to perturbations of variational inequalities, it should be pointed out that in our setting one can immediately obtain results on continuous dependence, perturbations, and approximations of the complementarity problem, using the results of Mosco [4].

\section{REFERENCES}

1. S. Karamardian, The nonlinear complementarity problem with applications. I, II, J. Optimization Theory Appl. 4 (1969), 87-98; 167-181. MR 40 \#5261.

2. T. Kato, Demicontinuity, hemicontinuity and monotonicity, Bull. Amer. Math. Soc. 70 (1964), 548-550; ibid. 73 (1967), 886-889. MR 29 \#501; MR 38 \#6411.

3. G. J. Minty, On a "monotonicity" method for the solution of nonlinear equations in Banach spaces, Proc. Nat. Acad. Sci. U.S.A. 50 (1963), 1038-1041. MR 28 \#5358.

4. U. Mosco, Consequence of convex sets and of solutions of variational inequalities, Advances in Math. 3 (1969), 510-585.

School of Industrial and Systems Engineering, Georgia Institute of Technology, Atlanta, Georgia 30332

School of Mathematics, Georgia Institute of Technology, Atlanta, Georgia 30332

Mathematics Research Center, University of Wisconsin, Madison, Wisconsin 3706 